\title{
Flare Activity in Algol-type Binaries
}

\author{
Izold Pustylnik \\ Institute of Astrophysics and Atmospheric Physics, Toravere, EE2444, Estonia
}

Two sites of flare activity have been identified in Algol-type binaries. One is associated with the shock region from the accretion on B-A type primary, another, predominant one is apparently connected with coronal mass ejections (CME) from the late-type, Roche lobe filling secondary. The relative contribution from both sources of activity is still to be found. According to Peters \& Polidan (1984), a moderately hot $\left(10^{5} \mathrm{~K}\right)$ and low density $n_{\mathrm{e}} \simeq 10^{9} \mathrm{~cm}^{-3}$ high turbulent accretion region (HTAR) surrounding the hot component has been discovered. Typical dimensions of HTAR agree with the scale height of the X-ray source $H_{\mathrm{X}} \simeq 1.2 \cdot 10^{11} \cdot T_{7} \mathrm{~cm}$ (Harnden et al 1977), where $T_{7}$ is the temperature in units of $10^{7} \mathrm{~K}$. But the decay time of the HTAR, comparable to the orbital period, more favourably agrees with the idea of CME as deduced from Ginga observations of X-ray flares on Algol (Stern et al. 1992). One solution of the controversy as to the contribution from HTAR would be to concentrate on X-ray observations of systems like $u \mathrm{Her}$ and $\mathrm{U} \mathrm{CrB}_{\mathrm{r}}$, where the secondary is possibly earlier than F0 (thus, is not expected to possess an extensive convection zone) and on the other hand on systems like U Cep, where during periods of high activity $\dot{M} \simeq 10^{-6} M_{\odot}$ per year. Thus, the accretion luminosity must be at least by two orders of magnitude higher than in Algol. At present the analysis of X-ray flares is based upon the solar type CME assuming different scenarios of radiative and/or conductive cooling (see Stern et al. 1992). However, the current models of CME do not take into account the binary nature of the flaring object.

We shall consider now a model of an anisotropic wind in a moderately close binary system where the wind from one component overwhelms the wind from the other. Our purpose is to estimate the effects of the anisotropy due to the displacement of a sonic point caused by the gravitational attraction of a companion. For a stationary flow in a radially expanding wind, and ignoring Coriolis forces

$$
\frac{1}{2}\left(1-\frac{u_{\mathrm{s}}^{2}}{u^{2}}\right) \frac{\mathrm{d} u^{2}}{\mathrm{~d} R}=\frac{2}{R} u_{\mathrm{s}}^{2}-\frac{\mathrm{d} u_{\mathrm{s}}^{2}}{\mathrm{~d} R}-\frac{\mathrm{d} \Phi}{\mathrm{d} R} .
$$

Here $u$ is the velocity of gas, $u_{\mathrm{g}}$ is the local sound velocity and $\Phi$ is the gravitational potential effectively reduced by radiation pressure both in continuum and in spectral lines. At the sonic point

$$
R=R_{\mathrm{s}}, u=u_{\mathrm{s}}, \quad \frac{2}{R_{\mathrm{s}}} u_{\mathrm{s}}^{2}-\left.\frac{2 k}{m} \frac{\mathrm{d} T}{\mathrm{~d} \mathrm{R}}\right|_{\mathrm{s}}-\left.\frac{\mathrm{d} \Phi}{\mathrm{d} R}\right|_{\mathrm{s}}=0 .
$$


For the evaporative wind we use the condition of conservation of mass flux $J=J_{0} \exp \left(-\Phi_{\mathrm{s}} / k T_{\mathrm{s}}\right) r^{2}$ along the streamline. We estimate the rate of anisotropy by assuming $T_{\mathrm{s}}=T_{\mathrm{s} 0}+(\mathrm{d} T / \mathrm{d} r) \Delta r_{\mathrm{s}}$, where $\mathrm{d} T / \mathrm{d} r$ is taken from Eq. (2) and $\Delta r_{\mathrm{s}}$ is the displacement of the sonic point. We assume $T=T_{\mathrm{s}}\left(r_{\mathrm{s}} / r\right)^{m}$ to estimate $\Delta r_{\mathrm{g}}$. Let us consider now a case of a plane-parallel thin conduction zone. The equation of heat conduction with the energy loss by radiation is

$$
\frac{\mathrm{d}}{\mathrm{d} r}\left(K_{0} T^{5 / 2} \frac{\mathrm{d} T}{\mathrm{~d} r}\right)=j_{0} n_{\mathrm{e}}^{2},
$$

where $K_{0}$ is rather insensitive to the density and the temperature (Hearn 1975). Substituting (2) for the temperature gradient into (3) one can estimate the angular dependence of the emission measure $j_{0} n_{\mathrm{e}}^{2}$. The results are indicated in Fig. 1 for some values of $\chi=G\left(M_{1}+M_{2}\right) / k T$ ( $\mu=1$ corresponds to the direction towards the centre of the accretor). The mass flux $J(\mu)$ also turns out to be directionally dependent by a factor of 3-5 being maximum for $\mu=0.6-0.7$. Thus, the anisotropy of the wind should affect the standard picture of the shock region (Richards 1992). Secondly, phase variations of $\mathrm{d} T / \mathrm{d} \mathrm{r}$ stemming from the binary nature of the source should be taken into account in future analysis.

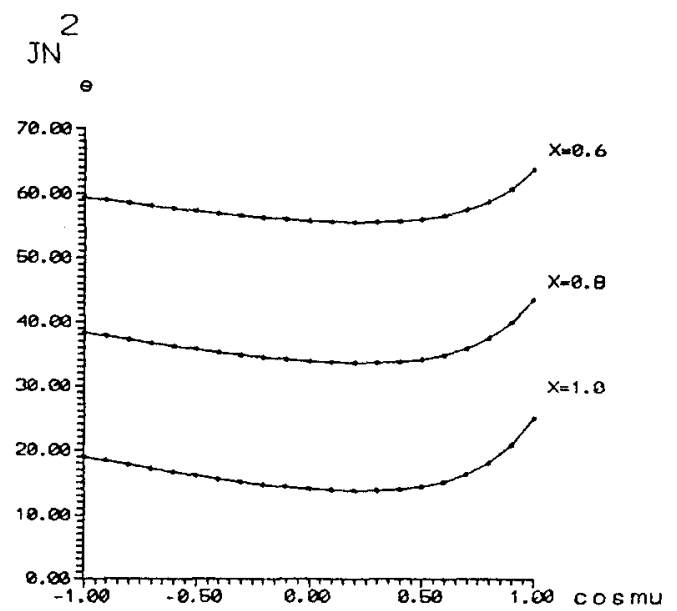

Fig. 1. An example of the angular dependence of the emission measure $j_{0} n_{e}^{2}$ for $\chi=0.6,0.8,1.0, \chi$ being the ratio of the escape velocity to the gas velocity at the sonic point, $\Gamma_{\mathrm{s}}=0.9,\left(\Gamma_{\mathrm{b}}\right.$ being the ratio of radiation pressure to the gravitational force of the mass losing component), mass ratio $q=1, r_{\mathrm{s}}=0.3, \mu=1$ corresponds to the direction towards the centre of the companion star.

\section{References}

Harnden Jr. F.R. , Fabricant D., Topka K., et al., 1977, ApJ 214, 418

Hearn A.G., 1975, A\&A 40, 355

Peters G.I., Polidan R.S., 1984, ApJ 283, 745

Richards M., 1992, ApJ 387, 328

Stern R.A., Uchida Y., Tsunata S., Nagase F., 1992, ApJ 400, 321 